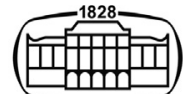

AKADÉMIAI KIADÓ

Acta Microbiologica et Immunologica Hungarica

67 (2020) 4, 209-215

DOI:

$10.1556 / 030.2020 .01181$

(C) 2020 Author(s)

RESEARCH ARTICLE

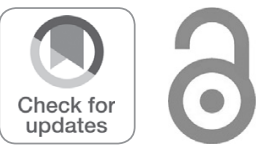

\title{
Detection of VIM, NDM and OXA-48 producing carbapenem resistant Enterobacterales among clinical isolates in Southern Hungary
}

MÁRIÓ GAJDÁCS ${ }^{1 *} \odot$, MARIANNA ÁBRÓK ${ }^{2}$, ANDREA LÁZÁR ${ }^{2}$, LAURA JÁNVÁRI ${ }^{3}$, ÁKOS TÓTH ${ }^{3}$, GABRIELLA TERHES ${ }^{3}$ and KATALIN BURIÁN ${ }^{2,4}$

\footnotetext{
${ }^{1}$ Department of Pharmacodynamics and Biopharmacy, Faculty of Pharmacy, University of Szeged, Eötvös utca 6., 6720 Szeged, Hungary

${ }^{2}$ Institute of Clinical Microbiology, Albert Szent-Györgyi Clinical Center, University of Szeged, Semmelweis utca 6., 6725 Szeged, Hungary

${ }^{3}$ Department of Bacteriology, Mycology and Parasitology, National Public Health Centre, Albert Flórián út 2-6., 1097 Budapest, Hungary

${ }^{4}$ Department of Medical Microbiology and Immunobiology, Faculty of Medicine, University of Szeged, Dóm tér 10., 6720 Szeged, Hungary
}

Received: March 22, 2020 - Accepted: April 4, 2020

Published online: December 1, 2020

\begin{abstract}
Infections caused by carbapenem-resistant Enterobacterales (CRE) present an important therapeutic problem, as there are limited number of effective therapeutic alternatives available. In this study, phenotypic and genotypic methods were used to characterize carbapenemase-production and other resistance-determinants (AmpC and ESBL-production, efflux pump-overexpression) in 50 isolates (Klebsiella spp. $n=35$, Escherichia coli $n=12$ and Enterobacter cloacae complex $n=3$ ) collected at the Albert Szent-Györgyi Clinical Center (University of Szeged) between 2014 and 2017. Minimum inhibitory concentrations of meropenem, sulfamethoxazole/trimethoprim, tigecycline, amikacin, moxifloxacin, colistin and fosfomycin were also determined. $24 \%$ of isolates were AmpC-producers, while $30 \%$ carried $b l a_{\text {CTX-M }}$ ESBL-genes. Carbapenemase-genes were detected in 18 (36\%) of the tested isolates: in 2 isolates $b l a_{N D M}$, in 6 isolates $b l a_{O X A-48-l i k e}$ and in 12 isolates, $b l a_{V I M}$ was detected by PCR. The species-distribution for isolates positive for carbapenemase-genes was the following: Klebsiella pneumoniae $n=11$, Klebsiella oxytoca $n=1$, E. coli $n=5$, E. cloacae complex $n=1$. Efflux pumpoverexpression based on the PA $\beta \mathrm{N}$-screening agar was shown in $n=3$ of the tested strains. In nine isolates (18\%), carbapenemase and ESBL-genes were detected simultaneously. Highest levels of resistance were noted for fosfomycin (74\%) and moxifloxacin (70\%), while all isolates were susceptible to colistin. Among applied phenotypic tests in this study the modified carbapenem inactivation method (mCIM) proved to be the most accurate one compared to that of PCR results.
\end{abstract}

\section{KEYWORDS}

carbapenem-resistant Enterobacterales, colistin, fosfomycin, carbapenemase, carbapenem inactivation method

\section{INTRODUCTION}

*Corresponding author. Tel.: +3662341330 E-mail: mariopharma92@gmail.com

Carbapenems, namely imipenem (introduced in 1985), meropenem (1996), ertapenem (2003) and doripenem (2007) are $\beta$-lactam-antibiotics with some of the broadest spectrum and bactericidal activity; they are effective in the therapy of infections caused by aerobic and anaerobic Gram-positive and Gram-negative pathogens (including non-fermenters, with the exception of ertapenem) [1,2]. Initially, carbapenems were exclusively used as monotherapy for life-threatening infections, however, after the rise and global spread of extended-spectrum 
$\beta$-lactamase (ESBL)-producing Enterobacterales, it has become an established clinical practice to use these agents in first-line empirical therapy $[2,3]$. Nevertheless, the extensive use of these agents resulted in the emergence of carbapenem-resistant Gram-negative strains [4]. From a clinical standpoint, carbapenem-resistant Gram-negative bacilli (CRGNB) present an important therapeutic problem, as there are limited number of safe and effective therapeutic alternatives available [5, 6]. Carbapenem resistance may develop through a variety of cellular mechanisms: alteration in membrane permeability and porin loss (e.g., $\Delta \mathrm{OmpK}$ in Klebsiella pneumoniae), overexpression of efflux pumps, changes in penicillin-binding proteins; however, the most prevalent mechanism of resistance is through the production of specific $\beta$-lactamases called carbapenemases, capable of hydrolyzing these antibacterial drugs [4, 7-10]. The differentiation of carbapenemase-producing carbapenem resistant Enterobacterales (CP-CRE; with Klebsiella spp. represented in highest numbers) from non-carbapenemase producing carbapenem resistant Enterobacterales (CRE) is of utmost importance, as the resistance-determinants of CP-CREs are readily transferable on plasmids or integrons, having a role in nosocomial outbreaks and global dissemination $[11,12]$. Based on sequence similarity, carbapenemases are classified into Ambler Class A e.g. KPC, SME, NMC-A, IMI, PER, GES, SFO, SFC and IBC), Class D (e.g. OXA-23 group, OXA-48-group) and Class B (e.g. VIM, GIM, SIM, NDM, IMP, IND, AIM, DIM and SPM) enzymes; while the first two groups consist of serine- $\beta$-lactamases, the members of the latter group are exclusively metallo- $\beta$-lactamases [13, 14]. Different carbapenemases have varying epidemiological significance around the world and in Europe, based on their origins and dissemination levels: ST258 $b l a_{\mathrm{KPC}}$ most likely have spread to Europe from the United States, while ST512 $b l a_{\mathrm{KPC}} \mathrm{s}$ are suggested to have spread to Europe from Israel [15]; $b l a_{\mathrm{VIM}-1}$ emerged in Greece and $b l a_{\mathrm{OXA}-48}$ emerged in Turkey and spread to Europe through the Mediterranean [16]. In Hungary, the first published case of carbapenemaseproduction in an Enterobacterales clinical isolate was reported in a KPC-2 and SHV-12-producing K. pneumoniae; the isolate originated from a patient, who has been previously hospitalized in Greece and caused a local outbreak in the northeastern region of the country $[16,17]$.

Various laboratory methods are available for the detection of CP-CRE strains, although the methods-of-choice are highly dependent on the epidemiological situation and the economic possibilities of the given healthcare settings [18]. Molecular methods, such as polymerase chain reaction (PCR) and whole-genome sequencing (WGS) are considered as the gold standard for detection of carbapenemase genes, nevertheless, their high laboratory costs make them unsuitable for many smaller, low-resource laboratories [19]. In addition, molecular methods are only capable of detecting already known resistance-determinants. Thus, carbapenemase-detection is usually carried out as a step-wise process: if carbapenemase-production is suspected based on the results of antimicrobial susceptibility testing, phenotypic screening (e.g., chromogenic media) or confirmatory tests (detection of diffusible carbapenemases, carbapenemase-inhibitor assays, assessment of carbapenem hydrolysis activity by measuring $\mathrm{pH}$-change or spectrophotometry), followed by molecular methods, if available [18-21]. Additionally, the use of lateral flow assays, microarray technology and matrix-assisted laser desorption/ ionization time-of-flight mass spectrometry for carbapenemase-detection has also been described [22, 23].

In the suspicion of CP-CRE arises, rapid screening and verification is essential, both to ensure appropriate therapy to the affected patients and to allow for infection control measures to take place $[16,18]$. Knowledge on the local epidemiology of each healthcare institution regarding carbapenemase-producing strains is of utmost importance, as this may aid the selection of the ideal methodology for their screening $[16,18]$. The aim of our study was the characterization of Enterobacterales strains suspected of carbapenemase-production in a low-prevalence setting in Hungary, through the use of various phenotypic and genotypic methods.

\section{MATERIALS AND METHODS}

\section{Bacterial strains, identification, inclusion criteria}

Fifty $(n=50)$ Enterobacterales strains, isolated from clinical samples between 2014 and 2017 at the Institute of Clinical Microbiology (Albert Szent-Györgyi Clinical Center, Szeged, Hungary) were included in this study. The identification of these isolates was carried out using MALDI-TOF MS. Mass spectrometry was performed by Microflex LT MALDI Biotyper (Bruker Daltonics, Bremen, Germany) instrument, using the MALDI Biotyper RTC 3.1 software and the MALDI Biotyper Library 3.1 for the spectrum analysis (Bruker Daltonics, Germany). The sample preparation, methodology, and technical specification of the MALDI-TOF MS measurements were described elsewhere [24]. Inclusion of these strains was based on the screening criteria recommended by the ESCMID/ EUCAST guidelines (meropenem disk diameter $<28 \mathrm{~mm}$ ) [25].

\section{Minimum inhibitory concentrations (MICs) of meropenem and ancillary antibiotics}

MICs of meropenem (MER), sulfamethoxazole/trimethoprim (SXT), tigecycline (TIG), amikacin (AMK) and moxifloxacin (MOX) were determined by E-tests (Liofilchem, Roseto degli Abruzzi, Italy) on Mueller-Hinton agar plates (Oxoid, Basingstoke, UK). MIC determination for colistin (COL) was carried out using the broth microdilution method in cation-adjusted Mueller-Hinton broth (MERLIN Diagnostika, Berlin, Germany) [24, 26]. MIC determination for fosfomycin (FOS) was carried out using the agar dilution method, on Mueller-Hinton agar plates supplemented with $25 \mathrm{mg} / \mathrm{L}$ glucose-6-phosphate and varying concentrations of FOS. The interpretation of the results was based on EUCAST Clinical Breakpoints v.9 (http://www.eucast.org). In case of isolates other than E. coli, epidemiological cut-off values were used for tigecycline $(\mathrm{MIC} \leq 1 \mathrm{mg} / \mathrm{L}$ as susceptible, MIC $>1 \mathrm{mg} / \mathrm{L}$ as resistant) [26]. 


\section{Phenotypic detection of AmpC- $\beta$-lactamases and ESBL-enzymes}

The strains included in the study were screened for AmpC- $\beta$ lactamase-production by cefoxitin disks (30 $\mu \mathrm{g}$; Oxoid, Basingstoke, UK) [27]. Phenotypic verification of AmpC- $\beta$ lactamase and ESBL-production was carried out using AmpCESBL Detection Set (MAST Diagnostica, Reinfeld, Germany) and VITEK 2 Compact ID/AST (bioMérieux, Marcy-l'Étoile, France), according to the manufacturer's instructions.

\section{Phenotypic screening and verification of carbapenemase-production}

For phenotypic screening of carbapenemase-production, the chromID CARBA SMART agar (CARB/OXA; bioMérieux, Marcy-l'Étoile, France) was used; this medium consists of a nutrient base, combining different peptones, three chromogenic substrates enabling the detection of specific metabolic enzymes for E. coli, Klebsiella spp., Enterobacter spp., Serratia spp. and Citrobacter spp., in addition to a proprietary mixture of antibiotics, favoring the selective growth of carbapenemase-producers [28]. The phenotypic verification of carbapenemase-production was carried out using the modified cloverleaf (or Hodge) test and the modified carbapenem inactivation method (mCIM), as previously described $[29,30]$. In both assays, meropenem disks (10 $\mu \mathrm{g}$; Oxoid, Basingstoke, UK) were utilized and E. coli ATCC 25922 was used as an indicator organism.

\section{Phenotypic detection of efflux pump-overexpression}

The effects of phenylalanine-arginine $\beta$-naphthylamide $(\mathrm{PA} \beta \mathrm{N})$, on the MICs of meropenem were detected using the agar dilution method described previously [27]. During the experiments, the concentration of $\mathrm{PA} \beta \mathrm{N}$ was $40 \mu \mathrm{g} / \mathrm{mL}$ in the agar base. Two-fold decrease in the MICs of meropenem in the presence of PA $\beta \mathrm{N}$ (a compound with well-known efflux-pump inhibitory activity [30]), compared to the MIC values without the inhibitor was considered as positivity for efflux pump overexpression.

\section{Molecular detection of ESBL and carbapenem- resistance genes}

Molecular detection of resistance genes encoding for ESBLs and carbapenemases was carried by multiplex PCR with previously described protocols [31-33].

\section{Statistical analyses}

Based on the data provided during our experiments, sensitivity, specificity, positive and negative predictive values (SN, SP, PPV and NPV, respectively) were calculated for each test, as described previously [34].

\section{RESULTS}

\section{Sample types and species composition of the isolates}

During the 4-year study period, 50 Enterobacterales isolates with meropenem disk diameters under $28 \mathrm{~mm}$ were detected: the majority $(n=35)$ of these isolates were Klebsiella spp. (K. pneumoniae: $n=34$, Klebsiella oxytoca: $n=$ $1)$, while E. coli $(n=12)$ and E. cloacae complex $(n=3)$ were also suspected for carbapenemase-production in lower numbers. These isolates originated from the following samples: urine: $n=19$ (midstream urine: $n=14$, catheterspecimen urine: $n=5$ ), feces: $n=14$, tracheal aspirate: $n=$ 7, abscess: $n=4$, aerobic wound culture and bile: $n=3$, respectively.

\section{MICs of tested antibiotics}

The MICs of tested antibiotics, including $\mathrm{MIC}_{50}, \mathrm{MIC}_{90}$ values, MIC ranges and the percentage of resistant isolates are presented in Table 1 . The highest levels of resistance were observed for FOS ( $n=37,74 \%)$, followed by MOX ( $n$ $=35,70 \%$ ), while non-susceptibility of the isolates for SXT and AMK were around 50\%. All tested isolates were susceptible to colistin, with MIC values ranging between 0.25 and $1 \mathrm{mg} / \mathrm{L}$. Based on EUCAST breakpoints, $n=14$ (28\%) of isolates showed MICs above the resistance breakpoint (8 $\mathrm{mg} / \mathrm{L}$ ) for meropenem, with MICs ranging between 0.25 and $32 \mathrm{mg} / \mathrm{L}$. Highest MICs were observed for the $b l a_{\mathrm{NDM}}$ and several bla $a_{\mathrm{VIM}}$-expressing strains, while in $n=4(8 \%)$ of cases, strains carrying bla $a_{\text {OXA48-like enzymes showed MICs in }}$ low-to-moderate range $(0.5-1 \mathrm{mg} / \mathrm{L})$ (Table 1.).

\section{Phenotypic and genotypic detection of resistance- determinants}

Based on the cefoxitin screening disk, 14 out of 50 isolates (28\%) were suspected of AmpC $\beta$-lactamase-production, while based on the AmpC-ESBL Detection Set, $n=12$ (24\%) of these isolates were phenotypically verified as AmpCproducers. The AmpC-ESBL Detection Set showed ESBLproduction in $n=15$ (30\%) isolates; in line with phenotypic results, $b l a_{\mathrm{CTX}-\mathrm{M}^{-}}$-type ESBL-genes were detected in all of these isolates. In $n=6(12 \%)$ of isolates, AmpC and ESBL co-production was verified, both by phenotypic and genotypic methods.

Using the modified cloverleaf test, 20 isolates showed positive results for carbapenemase-production, while this number was $n=19$ for the chromID CARBA SMART agar and $n=18$ for the mCIM assay. Carbapenemase-genes were

Table 1. MIC values of meropenem and ancillary antibiotics on the tested bacterial strains

\begin{tabular}{lcccc}
\hline & $\begin{array}{c}\text { Resistant } \\
\text { strains (n, } \\
\%)\end{array}$ & $\begin{array}{c}\text { MIC } \\
\text { range } \\
(\mathrm{mg} / \mathrm{L})\end{array}$ & $\begin{array}{c}\mathrm{MIC}_{50} \\
(\mathrm{mg} / \mathrm{L})\end{array}$ & $\begin{array}{c}\mathrm{MIC}_{90} \\
(\mathrm{mg} / \mathrm{L})\end{array}$ \\
\hline Meropenem & $14(30 \%)$ & $0.25-32$ & 8 & 16 \\
Amikacin & $24(48 \%)$ & $1-16$ & 4 & 16 \\
Colistin & $0(0 \%)$ & $0.25-1$ & 0.5 & 1 \\
Fosfomycin & $37(74 \%)$ & $1-64$ & 16 & 64 \\
Moxifloxacin & $35(70 \%)$ & $0.064-1$ & 0.25 & 1 \\
Sulfamethoxazole/ & $24(48 \%)$ & $0.064-16$ & 2 & 8 \\
$\quad$ trimethoprim & $16(32 \%)$ & $0.064-1$ & 0.25 & 1 \\
Tigecycline & & & & \\
\hline
\end{tabular}


detected by PCR in $n=18$ (36\%) of the tested isolates: in 2 strains $b l a_{N D M}$, in 6 strains were bla $a_{O X A-48 \text {-like }}$ and in 12 $b l a_{V I M}$ was detected. In nine isolates $(18 \%)$, carbapenemase and ESBL-genes were detected simultaneously ( $b l a_{\mathrm{VIM}}$ and bla $a_{\text {CTX-M }}$ co-detection in $n=7$ cases, bla $a_{\text {OXA-48-like }}$ and bla $a_{\text {CTX-M }}$ co-detection in $n=2$ cases). The species-distribution for isolates positive for carbapenemase-genes was the following: $K$. pneumoniae $n=11, K$. oxytoca $n=1$, E. coli $n$ $=5$, E. cloacae complex $n=1$. No ESBL or carbapenemaseencoding genes or phenotypic AmpC-production were detected in $n=5(10 \%)$ of isolates. Efflux pump-overexpression based on the $\mathrm{PA} \beta \mathrm{N}$-screening agar was shown in $3(6 \%)$ of the tested strains only: one isolate was ESBLpositive, one was an AmpC-producer, while in one isolate, no other resistance determinant could be detected.

Based on the comparative analysis of phenotypic and genotypic carbapenemase detection methods (considering molecular methods as the gold standard), three isolates presented false-positive (17/20 were true positives) and one presented as false-negative (29/30 were true negatives), while the chromogenic screening agar showed once false-positive (18/19 were true positives). False positivity was noted in case of an AmpC-producer (E. coli), an AmpC-ESBL co-producer (E. cloacae complex) and an AmpC-efflux pump overexpresser (K. pneumoniae); in contrast, the strain presenting as false-negative in both methods was a $b l a_{\text {OXA-48-like-pro- }}$ ducer. The mCIM method was completely in line with the results of genotypic testing (zero false positives or false negatives). Determination of the predictive power of the individual screening and verification tests is presented in Table 2.

\section{DISCUSSION}

Members of the Enterobacterales order are common causes of both community-acquired and nosocomial infections, including bloodstream-infections, lower respiratory tract infections and urinary tract infections [35]. Carbapenems have become essential components of therapy in infections caused by extended-spectrum cephalosporin resistant strains, especially for vulnerable patient groups, such as children, pregnant women and the elderly [1-4,36]. Although intrinsic resistance to carbapenems has been previously described in Stenotrophomonas maltophilia (conferred by the metallo- $\beta$-lactamase L1), acquired

Table 2. Calculated predictive power of the individual screening and verification tests employed the study

\begin{tabular}{lcccc}
\hline & $\begin{array}{c}\text { Sensitivity } \\
(\%)\end{array}$ & $\begin{array}{c}\text { Specificity } \\
(\%)\end{array}$ & $\begin{array}{c}\text { PPV } \\
(\%)\end{array}$ & $\begin{array}{c}\text { NPV } \\
(\%)\end{array}$ \\
\hline $\begin{array}{l}\text { Modified cloverleaf } \\
\text { (Hogde) test }\end{array}$ & 94.7 & 91.4 & 85.7 & 96.9 \\
chromID CARBA \\
$\quad 100$ & 96.9 & 94.7 & 100 \\
$\begin{array}{l}\text { SMART } \\
\text { Modified carbapenem } \\
\text { inactivation method } \\
(\mathrm{mCIM})\end{array}$ & 100 & 100 & 100 & 100 \\
\hline
\end{tabular}

carbapenem-resistance (through mutational events or via horizontal gene transfer) has emerged as a significant clinical problem in the last 15-20 years [37, 38]. The spread of carbapenemresistant strains in nosocomial settings, where MDR Gramnegative bacteria are already endemic, is especially daunting [39]. These pathogens may acquire resistance determinants, conferring resistance for multiple antibiotic classes, leading to virtually untreatable infections [40]. CP-CRE species have now been reported worldwide; carbapenemases have mainly been found in $K$. pneumoniae, and to a much lesser extent in E. coli and other members of the Enterobacterales order [41]. Based on estimation of the Centers for Disease Control and Prevention (CDC), carbapenem-resistant Enterobacterales species may be causative agents in up to 9,000 infection and 600 excess deaths peryear in the US [42]. Similarly, the Burden of Antimicrobial Resistance Collaborative Group estimated that in Europe, around 16,000 infections and 2000 attributable deaths occurred in 2015 due to carbapenem-resistant K. pneumoniae [43]. The distribution of carbapenemase-producing isolates worldwide has been recently summarized by the Center for Disease Dynamics, Economics and Policy database: according to this database, $b l a_{\text {OXA-48-like }}$ is endemic in Africa, $b l a_{\mathrm{OXA}-48-\text { like, }} b l a_{\mathrm{NDM}}, b l a_{\mathrm{KPC}}$ and $b l a_{\mathrm{IMP}}$ in Asia, $b l a_{\mathrm{KPC}}$ in the American Continent and $b l a_{\mathrm{OXA}-48 \text {-like, }} b l a_{\mathrm{VIM}}$, $b l a_{\mathrm{KPC}}$ and $b l a_{\mathrm{IMP}}$ in Europe [44]. Based on the data of the ECDC Surveillance on Antimicrobial Resistance in 2017 and 2018, Greece had the highest levels of carbapenem-resistant isolates (64.7 and $63.9 \%$ for K. pneumoniae and 1.6 and $2.0 \%$ for E. coli), followed by Italy (26.8 and $29.7 \%$ for K. pneumoniae) and Romania (22.5 and $29.5 \%$ for K. pneumoniae) [45]; this is not surprising, as Greece has been considered the epicenter of CPCRE strains on the continent, where the spread of these strains usually showed a south-to-north gradient $[37,38]$. In Hungary, the ratio of carbapenem-resistant $E$. colistrains was $<0.1 \%$ for both years, while for K. pneumoniae, the percentage of relevant isolates was still very low (0.2 and $0.3 \%$, respectively) [23, 42$]$.

The costs, turnaround-time, accuracy, the information provided by these screening and phenotypic tests and the expected output (clinical, local or regional epidemiology or national/international surveillance) all need to be taken into consideration when opting for a carbapenemase-detection strategy [46]. While some test may be suitable for local microbiology laboratories for rapid therapeutic decisions, others will only be available to reference laboratories. The phenotypic detection of carbapenemases is hindered by their diversity, the pronounced differences in hydrolytic ability and their frequent co-occurrence with other resistance mechanisms influencing $\beta$-lactam resistance $[47,48]$. For example, in strains with ESBLs or AmpC-hyperproduction, coupled with porin loss and/or efflux pump-overexpression, a similar phenotype may be observed to carbapenemase-production in phenotypic tests $[47,48]$. On the other hand, some carbapenemase-enzymes do not confer high MICs to the isolates, therefore there is a risk that they will be missed by disk diffusion/gradient tests [49]. In addition, the local epidemiology of carbapenemases also influences the phenotypic tests employed, based on the sensitivity of these tests for the specific enzymes [16, 18]. The advantage of inhibitor tests is that they may be used to distinguish between different types (i.e. serine or metallo- $\beta$ - 
lactamases) or enzymes [47, 48]. The poor sensitivity and specificity of the modified cloverleaf (Hodge) test has been documented previously: false-positive results are common in other $\beta$-lactamase (ESBL or AmpC) producers, with or without porin mutations or efflux pumps, while some types of carbapenemases ( $b l a_{\mathrm{OXA}-48 \text {-like }}$ and $b l a_{\mathrm{NDM}^{-}}$-types, although the introduction of $\mathrm{Zn}^{2+}$ to the culture media results in increased sensitivity for the latter type) have shown false-negative results [49]. Several modifications and adjustments have been published with the aim of improving the detection parameters of this assay [50]. The advantage of screening media (e.g., chromID CARBA SMART utilized in this study) is that they are ready-to-use, assistant-friendly and ease of interpretation and sensitivity/specificity of this method has been demonstrated to be around $96-100 / 97-100 \%$ in several studies [26, 51]; nevertheless, the verification of culture results with additional phenotypic tests is recommended. The modified carbapeneminactivation method (mCIM) is easy-to-perform and the panels can be prepared in-house; this assay has also demonstrated sensitivities/specificities $\geq 98 \%$ in many publications $[27,52]$. The use of the CarbaNP confirmatory test (and the numerous iterations of this method) is becoming more and more widespread: the method is based on the direct detection of carbapenem hydrolysis, and similarly to the mCIM test, both commercial and in-house panels are available to use [53]. Nonetheless, molecular methods (predominantly single or multiplex PCR-based, with results in 2-6 hours) remain the reference for identification and differentiation of carbapenemases and this may be followed by sequencing, if available [18, 54]. Their cost and the need for trained technicians however, are significant drawbacks.

While there has been some developments in the introduction of new $\beta$-lactam/non-boronic or boron-based $\beta$-lactamase-inhibitor combinations (e.g., ceftolozane/tazobactam, ceftazidime/ avibactam, meropenem/vaborbactam) in therapy for Class A carbapenemases, we currently do not possess adequate $\beta$-lactamtherapy for metallo- $\beta$-lactamase-producing (Class B) strains [55]; these $\mathrm{Zn}^{2+}$-dependent enzymes are inhibited by metal chelators, such as EDTA or dipicolinic acid, but no clinicallyrelevant metallo- $\beta$-lactamase-inhibitor has been identified thus far [56]. An additional therapeutic concern is the possibility of strains possessing more than one type of carbapenemase, eliminating $\beta$-lactams as potential therapeutic options [56]. Carbapenemases in Enterobacterales are mostly plasmid-encoded and are often associated with resistance-determinants to other antibiotics, especially for fluoroquinolones and aminoglycosides [57]. Colistin is nephrotoxic and neurotoxic polycationic peptide, which is usually considered as a last-resort agent in MDR Gram-negative infections [58]; in our study, all of the tested isolates were susceptible. Nonetheless, the increasing frequency of reports on colistin-resistance in Enterobacterales and non-fermenters is a serious concern; in the EuSCAPE Survey (European survey of carbapenemase-producing Enterobacteriaceae) $28.3 \%$ of CRE isolates were also colistin-resistant [59]. TIG resistance was observed in $32 \%$ of isolates; this agent has been introduced with great promise in the therapy of MDR Gram-negative infections, however, the black-box warning issued by the FDA (due to increased overall mortality in TIG- treated patients) and low tissue penetration and serum-levels, it did not live up to the expectations. The use of intravenous FOS in the therapy of MDR Gram-negative bacteria has been extensively reported as a promising alternative [60]; nevertheless, 74\% of our isolates tested were resistant to this antibiotic. In a Turkish study, the susceptibilities of CP-CRE isolates were assessed and the authors reported $76.2 \%$ of isolates as COL-resistant and $67.4 \%$ as FOS-resistant [26]. On the other hand, the literature is scarce on the relevance of SXT-therapy for CP-CRE infections: in our study, $48 \%$ of isolates were still susceptible to SXT; in the CRACKLE- 1 cohort study in the US, $29 \%$ of pathogens in CPCRE infections were susceptible to SXT and one-third of these affected patients were successfully treated with the drug [61]. In contrast, in a survey involving $n=181$ CP-CRE isolates, Baran et al. showed that SXT-resistance in E. coli and K. pneumoniae was 61 and 55\%, respectively [62].CP-CRE isolates havevery few or no therapeutic options (depending on resistance rates to ancillary antibiotics) available. Thus, their optimal monitoring and rapid detection for therapeutic, infection control and epidemiological purposes is needed. The findings of our study indicate that out of the 50 suspected isolates, presence of carbapenemase-genes verified in 18 strains, and the phenotypic methods utilized (especially the chromogenic media for screening and mCIM for verification) were appropriate to use in our settings. Nevertheless, every laboratory and/or healthcare institution needs to pre-test various testing methods and consult the national reference laboratory to select for adequate detection methods for carbapenemase-producers.

Conflict of interest: The authors declare no conflict of interest, monetary or otherwise.

\section{ACKNOWLEDGMENTS}

The authors would like to thank the laboratory technicians of the Institute of Clinical Microbiology (University of Szeged) and Department of Bacteriology, Mycology and Parasitology (National Institute of Public Health) for their excellent assistance during the routine diagnostic work. M.G. was supported by the János Bolyai Research Scholarship (BO/00144/20/5) of the Hungarian Academy of Sciences. The research was supported by the ÚNKP-20-5SZTE-330 New National Excellence Program of the Ministry for Innovation and Technology from the source of the National Research, Development and Innovation Fund. Support from Ministry of Human Capacities, Hungary grant 20391-3/2018/FEKUSTRAT is acknowledged. M.G. would also like to acknowledge the support of ESCMID's “30 under 30" Award.

\section{REFERENCES}

[1] Papp-Wallace KM, Endimiani A, Taracila MA, Bonomo RA. Carbapenems: Past, Present, and Future. Antimicrob Agent Chemother 2011; 55: 4943-60. 
[2] El-Gamal MI, Brahim I, Hisham N, Aladdin R, Mohammed H, Bahaaeldin A. Recent updates of carbapenem antibiotics. Eur J Med Chem 2017; 131: 185-95.

[3] Frakking FNJ, Rottier WC, Dorigo-Zetsma W, van Hattem JMA, van Hees BC, Klutymans JAWJN, et al. Appropriateness of empirical treatment and outcome in bacteremia caused by extended-spectrum- $\beta$-Lactamase-producing bacteria. Antimicrob Agent Chemother 2013; 57: 3092-9.

[4] Codjoe FS, Donkor ES. Carbapenem resistance: a review. Med Sci 2018; 6: e1.

[5] Poole K. Pseudomonas Aeruginosa: resistance to the max. Front Microbiol 2011; 2: e65.

[6] van Duin D, Kaye KS, Neuner EA, Bonomo RA. Carbapenemresistant Enterobacteriaceae: a review of treatment and outcomes. Diagn Microbiol Infect Dis 2013; 75: 115-20.

[7] Meletis G. Carbapenem resistance: overview of the problem and future perspectives. Ther Adv Infect Dis 2016; 3: 15-21.

[8] Livorsi DJ, Chorazy ML, Schweizer ML, Balkende EC, Blevins AE, Nair R, et al. A systematic review of the epidemiology of carbapenem-resistant Enterobacteriaceae in the United States. Antimicrob Res Infect Control 2018; 7: e55.

[9] Karlowsky JA, Lob SH, Kazmierczak KM, Badal RE, Young K, Motyl MR, et al. In vitro activity of imipenem against carbapenemase-positive enterobacteriaceae isolates collected by the SMART global surveillance program from 2008 to 2014. J Clin Microbiol 2017; 55: 1638-49.

[10] Lee CS, Doi Y. Therapy of infections due to carbapenem-resistant gram-negative pathogens. Infect Chemother 2014; 46: 149-64.

[11] Lee CR, Lee HJ, Park SK, Kim YB, Jeong BC, Lee SH. Global dissemination of carbapenemase-producing Klebsiella pneumoniae: epidemiology, genetic context, treatment options, and detection methods. Front Microbiol 2016; 7: e895.

[12] Lutgring JD, Limbago BM. The problem of carbapenemase-producing-carbapenem-resistant-enterobacteriaceae detection. J Clin Microbiol 2016; 54: 529-34.

[13] Sahuquillo-Arce JM, Hernández-Cabezas A, Yarad-Auad F, Ibanez-Martínez E, Falomir-Salcedo P, Ruiz-Gaitán A. Carbapenemases: a worldwide threat to antimicrobial therapy. World J Pharmacol 2015; 4: 75-95.

[14] Queenan AM, Bush K. Carbapenemases: the versatile $\beta$-lactamases. Clin Microbiol Rev 2007; 20: 440-58.

[15] David S, Reuter S, Harris RS, Glasner C, Feltwell T, Argimon S, et al. Epidemic of carbapenem-resistant Klebsiella pneumoniae in Europe is driven by nosocomial spread. Nature Microbiol 2019; 4: 1919-29.

[16] Cantón R, Akóva M, Carmeli Y, Giske CG, Glupczynski Y, Gniadkowski M, et al. Rapid evolution and spread of carbapenemases among Enterobacteriaceae in Europe. Clin Microbiol Infect 2012; 18: 413-31.

[17] Tóth Á, Damjanova I, Puskás E, Jánvári L, Farkas M, Dobák A, et al. Emergence of a colistin-resistant KPC-2-producing Klebsiella pneumoniae ST258 clone in Hungary. Eur J Clin Microbiol Infect Dis 2010; 29: 765-9.

[18] Nordmann P, Gniadkowski M, Giske CG, Poirel L, Woodford N, Miriagou V, et al. Identification and screening of carbapenemproducing Enterobacteriaceae. Clin Microbiol Infect 2012; 18: $432-8$.
[19] Gupta V, Garg R, Kumaraswamy K, Datta P, Mohi KG, Chander J. Phenotypic and genotypic characterization of carbapenem resistance mechanisms in Klebsiella pneumoniae from blood culture specimens: a study from North India. J Lab Physicians 2018; 10: $125-9$.

[20] Baeza LL, Pfenningwerth N, Greissl C, Göttig S, Saleh A, Stelzer Y, et al. Comparison of five methods for detection of carbapenemases in Enterobacterales with proposal of a new algorithm. Clin Microbiol Infect 2019; 25: 1286.e9-e15.

[21] Cordeiro-Moura JR, Correra Fehlberg LC, Nodari CS, de Matos AD, Alves VO, Cayo R, et al. Performance of distinct phenotypic methods for carbapenemase detection: the influence of culture media. Diagn Microbiol Infect Dis 2020; 96: e114912.

[22] Rösner S, Kamalanabhaiah S, Küsters U, Kolbert M, Pfenningwerth N, Mack D. Evaluation of a novel immunochromatographic lateral flow assay for rapid detection of OXA-48, NDM, KPC and VIM carbapenemases in multidrug-resistant Enterobacteriaceae. J Med Microbiol 2019; 68: 379-81.

[23] Dortet L, Tandé D, de Briel D, Bernabeu S, Lasserre GG, Jousset $\mathrm{AB}$, et al. MALDI-TOF for the rapid detection of carbapenemaseproducing Enterobacteriaceae: comparison of the commercialized MBT STAR ${ }^{\circledR}$-Carba IVD Kit with two in-house MALDI-TOF techniques and the RAPIDEC ${ }^{\circledR}$ CARBA NP. J Antimicrob Chemother 2018; 73: 2352-9.

[24] Gajdács M, Ábrók M, Lázár A, Burián K. Comparative epidemiology and resistance trends of common urinary pathogens in a tertiary-care hospital: a 10-year surveillance study. Medicina 2019; 55: e356.

[25] EUCAST guideline for the detection of resistance mechanisms and specific resistances of clinical and/or epidemiological importance. Available from: http://www.eucast.org/fileadmin/src/media/PDFs/ EUCAST_files/Resistance_mechanisms/EUCAST_detection_of_ resistance_mechanisms_170711.pdf (Accessed: 22 March, 2020).

[26] Yildiz SS, Kaskatepe B, Simsek H, Sirgüzel FM. High rate of colistin and fosfomycin resistance among carbapenemase-producing Enterobacteriaceae in Turkey. Acta Microbiol Immunol Hung 2019; 66: 103-12.

[27] Khalili Y, Yekani M, Goli HR, Memar MY. Characterization of carbapenem-resistant but cephalosporin-susceptible Pseudomonas aeruginosa. Acta Microbiol Immunol Hung 2019; 66: 529-40.

[28] Vrioni G, Daniil I, Voulgari E, Ranellou K, Koumaki V, Ghirardi $\mathrm{S}$, et al. Comparative evaluation of a prototype chromogenic medium (ChromID CARBA) for detecting carbapenemase-producing Enterobacteriaceae in surveillance rectal swabs. J Clin Microbiol 2012; 50: 1841-6.

[29] Mathers AJ, Carroll J, Sifri CD, Hazen KC. Modified hodge test versus indirect carbapenemase test: prospective evaluation of a phenotypic assay for detection of Klebsiella pneumoniae Carbapenemase (KPC) in Enterobacteriaceae. J Clin Microbiol 2013; 51: 1291-3.

[30] Spengler G, Kincses A, Gajdács M, Amaral L. New roads leading to old destinations: efflux pumps as targets to reverse multidrug resistance in bacteria. Molecules 2017; 22: e468.

[31] Ellington MJ, Kistler J, Livermore DM, Woodford N. Multiplex PCR for rapid detection of genes encoding acquired metallo-betalactamases. J Antimicrob Chemother 2007; 59: 321-2.

[32] Poirel L, Walsh TR, Cuvillier V, Nordmann P. Multiplex PCR for detection of acquired carbapenemase genes. Diagn Microbiol Infect Dis 2011; 70:119-23. 
[33] Bradford PA. Extended-spectrum b-lactamases in the 21st century: characterization, epidemiology, and detection of this important resistance threat. Clin Microbiol Rev 2001; 14: 933-51.

[34] Yildiz SS, Kaskatepe B, Avcikücük H, Öztürk S. Performance of carbaNP and CIM tests in OXA-48 carbapenemase-producing Enterobacteriaceae. Acta Microbiol Immunol Hung 2017; 64: 9-16.

[35] Correa-Martinez CL, Idelevich EA, Sparbier K, Kostrzewa M, Becker K. Rapid detection of extended-spectrum $\beta$-lactamases (ESBL) and AmpC $\beta$-lactamases in Enterobacterales: development of a screening panel using the MALDI-TOF MS-based direct-ontarget microdroplet growth assay. Front Microbiol 2019; 10: e13.

[36] Karaiskos I, Giamarellou H. Carbapenem-sparing strategies for ESBL producers: when and how. Antibiotics 2020; 9: e61.

[37] Gajdács M, Urbán E. Prevalence and antibiotic resistance of Stenotrophomonas maltophilia in respiratory tract samples: A 10year epidemiological snapshot. Health Serv Res Manag Epidemiol 2019; 6: 2333392819870774.

[38] Lutgring JD, Zhu W, de Man TJB, Johannetsy JA, Anderson KF, Lonsway DR, et al. Phenotypic and genotypic characterization of Enterobacteriaceae producing Oxacillinase-48-like carbapenemases, United States. Emerg Infect Dis 2018; 24: 700-9.

[39] Pitout JDD, Nordmann P, Poirel L. Carbapenemase-producing Klebsiella pneumoniae, a key pathogen set for global nosocomial dominance. Antimicrob Agents Chemother 2015; 59: 5873-84.

[40] Abat C, Fournier PE, Jimeno MT, Rolain JM, Raoult D. Extremely and pandrug-resistant bacteria extra-deaths: myth or reality? Eur J Clin Microbiol Infect Dis 2018; 37: 1687-97.

[41] Livermore DM. Has the era of untreatable infections arrived? J Antimicrob Chemother 2009; 64: i29-36.

[42] CDC CRE Technical Information. Available from: https://www.cdc. gov/hai/organisms/cre/technical-info.html (Accessed: 11 February, 2020).

[43] Cassini A, Högberg LD, Plachouras D, Quattrocchi A, Hoxha A, Simonsen GS, et al. Attributable deaths and disability-adjusted life-years caused by infections with antibiotic-resistant bacteria in the EU and the European Economic Area in 2015: a populationlevel modelling analysis. Lancet Infect Dis 2019; 19: 55-66.

[44] Klein EY, Tseng KK, Pant S, Laxminarayan R. Tracking global trends in the effectiveness of antibiotic therapy using the Drug Resistance Index. BMJ Global Health 2019; 4: e001315.

[45] ECDC Surveillance Atlas of Infectious Diseases. Available from: https://atlas.ecdc.europa.eu/public/index.aspx? Dataset $=27 \&$ HealthTopic $=4$ (Accessed: 11 February, 2020).

[46] Workneh M, Yee R, Simner PJ. Phenotypic methods for detection of carbapenemase production in carbapenem-resistant organisms: what method should your laboratory choose? Clin Microbiol Newslett 2019; 41: 11-22.

[47] Bialvaei AZ, Kafil HS, Asgharzadeh M, Memar MY. Current methods for the identification of carbapenemases. J Chemother 2016; 28: 1-19.

[48] Bush K, Jacoby GA. Updated functional classification of $\beta$-lactamases. Antimicrob Agents Chemother 2010; 54: 969-76.
[49] Fattouh R, Tijet N, McGeer A, Poutanen SM, Melano RG, Patel $\mathrm{SN}$. What is the appropriate meropenem MIC for screening of carbapenemase-producing Enterobacteriaceae in low-prevalence settings? Antimicrob Agents Chemother 2015; 60: 1556-9.

[50] Girlich D, Poirel L, Nordmann P. Value of the modified Hodge test for detection of emerging carbapenemases in Enterobacteriaceae. J Clin Microbiol 2012; 50: 477-9.

[51] Pasteran F, Gonzalez LJ, Albornoz E, Bahr G, Vila AJ, Corso A. Triton Hodge test: improved protocol for modified hodge test for enhanced detection of NDM and other carbapenemase producers. J Clin Microbiol 2016; 54: 640-9.

[52] Liu Q, Liu L, Li Y, Chen X, Yan Q, Liu WE. Fecal carriage and epidemiology of carbapenem-resistant Enterobacteriaceae among hospitalized patients in a university hospital. Infect Drug Res 2019; 12: 3935-42.

[53] McMuller AR, Yarbrough ML, Wallace MA, Shupe A, Burnham CAD. Evaluation of genotypic and phenotypic methods to detect carbapenemase production in gram-negative Bacilli. Clinical Chem 2017; 63: 723-30.

[54] Pasteran F, Tijet N, Melano RG, Corso A. Simplified protocol for Carba NP test for enhanced detection of carbapenemase producers directly from bacterial cultures. J Clin Microbiol 2015; 53: 3908-11.

[55] Sugawara Y, Hagiya H, Akeda Y, Aye MM, Win HPM, Sakamoto $\mathrm{N}$, et al. Dissemination of carbapenemase-producing Enterobacteriaceae harbouring blaNDM or blaIMI in local market foods of Yangon, Myanmar. Sci Rep 2019; 9: 14455.

[56] Gajdács M, Albericio F. Antibiotic resistance: from the bench to patients. Antibiotics 2019; 8: e129.

[57] Palzkill T. Metallo- $\beta$-lactamase structure and function. Ann NY Acad Sci 2013; 1277: 91-104.

[58] Zhao Z, Lan F, Liu M, Chen W, Huang L, Lin Q, et al. Evaluation of automated systems for aminoglycosides and fluoroquinolones susceptibility testing for Carbapenem-resistant Enterobacteriaceae. Antimicrob Resist Infect Control 2017; 6: e77.

[59] Trebosc V, Gartenmann S, Tötzl M, Lucchini V, Schellhorn B, Pieren $M$, et al. Dissecting colistin resistance mechanisms in extensively drug-resistant Acinetobacter baumannii clinical isolates. mBio 2019; 10: e01083-19.

[60] Grundmann H, Glasner C, Albiger B, Aanersen DM, Tomlinson $\mathrm{CT}$, Andrasevic AT, et al. Occurrence of carbapenemase-producing Klebsiella pneumoniae and Escherichia coli in the European survey of carbapenemase-producing Enterobacteriaceae (EuSCAPE): a prospective, multinational study. Lancet Infect Dis 2017; 17: 153-63.

[61] Luterbach CL, Boshe A, Henderson HI, Cober E, Richter SS, Salata $\mathrm{RA}$, et al. The role of trimethoprim/sulfamethoxazole in the treatment of infections caused by carbapenem-resistant Enterobacteriaceae. Open For Infect Dis 2018; 6: ofy351.

[62] Baran I, Aksu N. Phenotypic and genotypic characteristics of carbapenem-resistant Enterobacteriaceae in a tertiary-level reference hospital in Turkey. Ann Clin Microbiol Antimicrob 2016; 15: e20.

Open Access. This is an open-access article distributed under the terms of the Creative Commons Attribution 4.0 International License (https://creativecommons.org/ licenses/by/4.0/), which permits unrestricted use, distribution, and reproduction in any medium, provided the original author and source are credited, a link to the CC License is provided, and changes - if any - are indicated. (SID_1) 